\title{
La socialización del consultor antropológico: de la práctica a los marcos normativos de la política pública
}

\section{The socialization of the anthropologist-consultant: from practice to the normative frameworks of policy}

\author{
Alejandro AGUDO SANCHÍZ \\ Departamento de Ciencias Sociales y Políticas \\ Universidad Iberoamericana. Ciudad de México \\ alejandro.agudo@ibero.mx
}

Recibido: 15 de abril de 2013

Aceptado: 2 de julio 2013

\section{Resumen}

Tanto si repudian como si defienden la llamada antropología aplicada, muchos antropólogos comparten lógicas similares con los integrantes de las comunidades profesionales de las políticas públicas. Este solapamiento se refleja en suposiciones sobre la separación y correlación jerárquica entre teoría y práctica, así como en diversos intentos por resolver la tensión entre lo general y lo particular. Estas coincidencias entre antropología y política, aunque también las diferencias epistemológicas que las separan, se muestran aquí a través del análisis de la evaluación de los impactos de un programa de desarrollo. Incluso antes de iniciar el trabajo de campo y regresar con sus descripciones etnográficas a las arenas institucionales de la política, los consultores antropológicos son disciplinados y socializados en el arte de reinterpretar los resultados de su investigación en términos del modelo del programa. La práctica produce y orienta a la política, si bien aquélla reside precisamente en los esfuerzos interpretativos de diversos participantes del desarrollo por sostener representaciones coherentes de la realidad frente a la contingencia de los acontecimientos.

Palabras clave: antropología y consultoría; políticas públicas; México; desarrollo; evaluación de impacto.

\begin{abstract}
Whether they reject or advocate so-called applied anthropology, many anthropologists share logics similar to those of the members of policy's professional communities. This overlap is reflected in assumptions about the separation of theory and "its" practice, as well as in different attempts to resolve the tension between the general and the particular. Both these similarities and the epistemological differences between anthropology and policy are outlined here through an account of a development programme's impact assessment. Even before beginning fieldwork and returning with their ethnographic descriptions to the institutional arenas of policy, anthropologist-consultants are disciplined and socialized into the art of reinterpreting their research results in terms of the programme's model. Practice produces and orders policy, yet such practice resides precisely in the interpretive efforts by diverse development practitioners to sustain coherent representations of reality against the contingency of events.
\end{abstract}


Keywords: anthropology and consultancy; public policy; Mexico; development; impact assessment.

Referencia normalizada: Agudo Sanchíz, A. (2013) La socialización del consultor antropológico: de la práctica a los marcos normativos de la política pública, en Revista de Antropología Social, 22: 177-198.

SUMARIO: 1. Introducción. 2. "Gemelos perversos": antropología y desarrollo social. 3. La evaluación de impacto como proceso de socialización del consultor. 4. Reflexiones finales. 5. Referencias bibliográficas

\section{Introducción}

A lo largo de las últimas dos décadas, de forma coincidente con la proliferación de etnografías de políticas ligadas a programas de desarrollo (Hobart, 1993a; Mosse, 2005; Mosse y Lewis, 2005; Viola, 2000), la antropología ha ido descubriendo a las políticas públicas como un "nuevo" campo de estudio para la disciplina (Shore y Wright, 1997) y a la consultoría como "una vertiente para el ejercicio profesional de los antropólogos" (García Espejel, 2012a). No obstante, como los propios Shore y Wright reconocen (1997), el estudio de las políticas —así como el trabajo en las mismas - conduce directamente a temas desde hace tiempo centrales en la antropología como la ideología, el conocimiento, el poder o el discurso. Otra cosa distinta es que, debido en parte a la sub-especialización de la antropología de las políticas públicas, ésta no haya aparecido habitualmente en las obras antropológicas sobre la política — con algunas excepciones (Nugent y Vincent, 2004; Sharma y Gupta, 2006; Gledhill, 2000)-.

Asimismo, la relación entre antropología y políticas públicas ha sido estrecha a lo largo de todo el siglo XX. Tan sólo el caso de México, donde vivo y trabajo, ofrece numerosos ejemplos de dicha relación, entre los que se encuentra el proyecto de desarrollo para la población del valle de Teotihuacan dirigido por el antropólogo Manuel Gamio entre 1917 y 1922 (véase Gamio, 1979). También destaca la participación de muchos otros practicantes de la disciplina en los proyectos de desarrollo industrial impulsados a mediados de siglo y, sobre todo, en la concepción y el empleo de las políticas indigenistas; incluso llegó a hablarse de una vinculación "orgánica" de la antropología al Estado mexicano a través de estas últimas (Lagarde, 1979). Ello alimentó las críticas lanzadas por la generación de antropólogos surgida del movimiento estudiantil de 1968, quienes vieron a las poblaciones indígenas del país como defensoras de su patrimonio cultural frente a las fuerzas modernizadoras representadas por las políticas asimilacionistas que sus antecesores habían fomentado (véase Warman, Nolasco, Bonfil, et. al., 1970).

Quizás sean estos antecedentes los que han contribuido, al menos en el caso mexicano, a la persistencia de sospechas y acusaciones de deshonestidad dirigidas en contra de antropólogos que trabajan para los organismos y programas de las 
políticas públicas. Estas perspectivas son a menudo sostenidas por aquellos otros integrantes de la disciplina considerados "puros" por no haberse "contaminado" en un mercado laboral ajeno a la academia y a la crítica independiente. A estos últimos se suele responder, sin embargo, que la negativa a realizar contribuciones prácticas en las políticas y otros ámbitos de trabajo — sustentada a menudo en buenos salarios y puestos académicos - no resulta éticamente menos problemática.

Una de las reacciones negativas más contundentes contra la sobrevaloración de lo académico y la obsolescencia de ciertas técnicas y temas de interés clásicos de la antropología, de nuevo en el caso de México, provino de una compilación de ponencias publicadas recientemente por la Universidad Autónoma de Querétaro (véase García Espejel, 2012a). Entre los autores de dichas ponencias se encuentran estudiantes frustrados por lo que perciben como una ausencia de "aproximaciones ... más prácticas al ejercicio profesional que nos espera allá afuera" (Martínez Romero, 2012: 52), así como de "herramientas necesarias para incorporarse a un mercado de trabajo ávido de antropólogos útiles, no de académicos" (Garibay, 2012: 224). Algunos de los textos aluden incluso a la necesidad de "vender la profesión" y hacerla más relevante a los ojos de los potenciales empleadores (Noroña, 2012), para lo cual recomiendan cambiar los vicios y actitudes "que supuestamente nos identifican como antropólogos: ser fachosos [de aspecto desaliñado], desadaptados, 'desmadrosos' y promiscuos, entre otras" (Martínez Romero, 2012: 45-46). En este sentido, una autora llega incluso a señalar las posibles consecuencias de nuestra empatía con los grupos marginados: "ahí reside la trampa: nos sentimos jodidos, buscamos el 'ser diferentes', ser parte de una minoría. Sin embargo, no podremos ayudar a los jodidos a salir de la jodidez desde la jodidez” (Vázquez-Mellado, 2012: 137).

Consistentes más en polémicas que en ensayos originales, estos textos tienen como telón de fondo un mercado laboral marcado por la precarización y saturación de puestos académicos, así como una comprensible desafección a los métodos y contenidos de una disciplina que, tal como se imparte y concibe en ciertos centros de enseñanza superior, aparece obsoleta y atrapada en los esencialismos y reduccionismos del pasado. En la manera en que se presentan, sin embargo, muchos de estos ataques corren el riesgo de reinstaurar por otra vía los estereotipos que pretenden combatir; algunos rayan incluso en un utilitarismo que lleva a sus autores a exigir que las escuelas de antropología aporten "la fuerza de trabajo para llenar los espacios que les corresponderían a los antropólogos aplicados", y a poner su afán en "incorporar a jóvenes antropólogos a esas instituciones que trabajan con los indígenas", así como a otras instancias y programas de agencias financieras nacionales o internacionales (Garibay, 2012: 226-227). El principal problema de estos resentimientos es que comparten con los académicos puros a los que van dirigidos una cuestionable dicotomía entre conocimiento para el entendimiento y conocimiento para la acción. Manifiesta en la persistente e incuestionada etiqueta de la "antropología aplicada", esta distinción constituye de hecho una de las formas institucionales adoptadas por la separación estricta entre teoría y práctica, como argumentaré más adelante. Muchos antropólogos trabajamos desde un variado espectro de posiciones simultáneas, gracias a las cuales somos capaces de combinar la participación activa con la distancia 
y la reflexión críticas. Éstas pueden quedar tergiversadas por la suposición de que la enseñanza de la investigación académica es útil sólo para sí misma y para la docencia (Vázquez-Mellado, 2012), o por el llamado a la práctica de la consultoría como vía privilegiada para "aplicar el conocimiento antropológico de manera concreta". Un aspecto adicional que estos supuestos ignoran es la proximidad que, de hecho, existe entre la antropología y los programas de desarrollo en cuanto a sus modos de ordenamiento discursivo e intentos por resolver tensiones entre lo general y lo particular (Yarrow, 2008). Son en parte los propios marcos de interpretación de los antropólogos, los cuales moldean nuestra experiencia y la transforman en datos y evidencia, los que hacen posible nuestra inserción en las comunidades profesionales de la política pública, así como en las relaciones sociales que inciden tanto en la producción de conocimiento como en la socialización de los consultores-antropólogos en el seno de las mismas. En lo que sigue pretendo desarrollar estas ideas empleando ejemplos de mi propia experiencia profesional en el ámbito de las políticas.

\section{2. "Gemelos perversos": antropología y desarrollo social}

El recelo antropológico hacia el desempeño extra-académico se ha sustentado a menudo en el riesgo de reforzar modelos etnocéntricos y disciplinarios de desarrollo social. En México llegó incluso a hablarse del "colonialismo interno" supuestamente practicado a través del indigenismo (González Casanova, citado en Aguirre Beltrán, 1992: 185, n. 168), en una suerte de reedición de aquella "antropología práctica" promovida de manera apasionada por Bronislaw Malinowski en la década de 1930 como fundamento para una ciencia de la política social colonial (Kuper, 2005). Justo cuando el infausto Proyecto Camelot parecía ya lejano en el tiempo, supimos del reclutamiento de antropólogos como asesores adscritos a unidades de combate del ejército de Estados Unidos en Afganistán e Irak, e incluso de la sustanciosa participación de algunos de esos antropólogos en la elaboración de un manual de contrainsurgencia militar estrenado en 2006 (González, 2007: 14; Houtman, 2006; Rohde, 2007). Sin duda, tales casos ilustran la cara más problemática de la también llamada engaged anthropology y, de paso, los matices semánticos menos edificantes de los términos "comprometida" o "involucrada". Sin llegar a estos extremos, no obstante, a algunos autores críticos como James Ferguson (1997) tampoco parecen faltarles ejemplos para hablar del desarrollo social como el "gemelo perverso" de la antropología, analizando el papel del primero en la constitución de la segunda con base en las mismas nociones evolucionistas del desarrollo que prevalecieron en la disciplina durante buena parte de los siglos XIX y XX'1

No obstante, el propio Ferguson (1994) y otros antropólogos críticos del desarrollo, influidos algunos también por Foucault (Escobar, 1995; Sachs, 1992), han sido cuestionados por presuponer efectos a partir de modelos de política, o incluso por sustituir resultados reales e imprevistos por los objetivos tácitos o explícitos de

${ }^{1}$ Podría argumentarse, sin embargo, que este tipo de perspectivas implica la habitual reducción de las políticas públicas —y el desempeño antropológico en las mismas - a proyectos de desarrollo. 
la planeación del desarrollo (Mosse, 2005). Paradójicamente, como apunta Mosse, esto no los hace tan distintos de los diseñadores y expertos de las políticas, aunque sustituyan el optimismo instrumental de estos últimos por suposiciones sobre el fracaso de las intervenciones del desarrollo o, peor, sobre el éxito de sus opresivos programas de poder y regulación. La política del desarrollo social puede sin duda analizarse como una forma de poder, pero las jerarquías de conocimiento y el control cognitivo, social o burocrático que pueda entrañar no se imponen a golpe de representaciones y discursos, sino que han de lograrse mediante la negociación, la colaboración y la socialización en lugares específicos (Li, 1999). Sostener lo contrario equivaldría a negar una vez más las posibilidades de la etnografía para iluminar tanto las contradicciones como los efectos reales de las políticas.

De manera irónica, entonces, la calidad de la antropología como gemelo perverso del desarrollo podría residir, en parte, en la tendencia a blindar los marcos normativos de la política social frente al impredecible mundo de la práctica y la experiencia. Al menos desde ciertas vertientes críticas, y por otras vías, se corre el riesgo de reinstaurar la lógica del ciclo de las políticas que asegura el poder de los planificadores sobre los "implementadores" y destinatarios, mediante prácticas y discursos que producen la aparente lógica y coherencia de las ideas de dichas políticas al tiempo que perpetúan el mito de su separación de los órdenes sociales que supuestamente gobiernan (véase Mitchell, 2002).

Lo que quizás sorprenda aún más es que, mediante sus llamados a la práctica antropológica como "aplicación" de conocimientos, sean los propios defensores del binomio antropología-consultoría los que también contribuyan a perpetuar la separación y correlación jerárquica entre ideas y objetos — con la contradicción adicional de invocar la puesta en práctica de investigaciones y enseñanzas académicas que previamente habían desdeñado por "inútiles", como también señalé anteriormente- . Junto con la relación entre antropología y políticas públicas, este reduccionismo viene de lejos en México, por lo que a los que lo sostienen no les vendría mal (re)leer uno de los capítulos reunidos en la Obra Polémica de Gonzalo Aguirre Beltrán. Éste percibía la separación entre investigación y acción como uno de los aspectos más rebatibles de los ataques que los antropólogos críticos de la generación del 68 dirigieron en su contra, como representante notorio de un indigenismo integracionista clásico tenido por colonialismo interno. En particular, Aguirre Beltrán rechazaba la acusación de que el indigenismo mexicano se hubiera apropiado sin más de los resultados de la investigación etnológica:

[U]na antropología aplicada derivada de una antropología teórica, si fuera posible, implicaría una separación entre lo teórico y lo práctico, esto es, significaría situar en terrenos diferentes a los etnólogos desinteresados que orientan sus investigaciones "por una problemática ajena a los objetivos de la política indigenista" y los ejecutores de los programas que se apropian "de los resultados de la etnología"; es decir, estaríamos negando un postulado indigenista que, en Teoría de la investigación intercultural, expresé diciendo: "investigación y acción son sólo fases de un mismo proceso polar” (Aguirre Beltrán, 1992: 200). 
Aguirre Beltrán ofrecía el ejemplo del ya mencionado proyecto de desarrollo para el valle de Teotihuacan, dirigido por Manuel Gamio y realizado por un equipo interdisciplinario cuyos integrantes hicieron investigación al tiempo que llevaban a cabo acciones para modificar la realidad de las poblaciones atendidas. El levantamiento de censos agrarios sirvió así para auxiliar a dichas poblaciones en la solicitud de dotaciones de tierras, mientras que el estudio de los cultivos llevó a los investigadores a sugerir métodos para mejorar e intensificar la producción agrícola y ganadera. Investigación y acción concurrieron de esta forma para generar un modelo de desarrollo regional — en lugar de simplemente "aplicar" dicho modelo- que pudiera extenderse a otras regiones:

Según es fácil advertir, el modelo de investigación-acción construido por Gamio
para alcanzar el desarrollo regional, no es simple aplicación de la teoría antropo-
lógica a un problema particular inmediato ni, por tanto, antropología aplicada tal y
como la entiende Medina [uno de los críticos del indigenismo], sino antropología
social genuina cuya idiosincrasia se funda en un proceso de recíproca influencia
entre teoría y práctica y que tiene como propósito deliberado modificar una realidad
indeseable (Aguirre Beltrán, 1992:202).

A pesar de su antigüedad, sin embargo, los argumentos sobre la inextricable relación entre el conocimiento y el orden social de su producción continúan soslayándose por varias razones. Por una parte, la visión del conocimiento como una serie de actividades constituidas mediante una historia cambiante de experiencias concretas suele llevarse mal con la política pública, cuyo principio fundamental descansa aun en la autonomía del Estado como agente interventor en la sociedad y como creador y regulador de dichas experiencias. Por otra parte, la cosificación del conocimiento y su separación de la práctica obedece a la necesidad de transferirlo y replicarlo a partir del diseño de programas de desarrollo y de sus evaluaciones de impacto. Si los científicos sociales no presentan sus resultados de esta forma, se socavan las bases de su empleo en la política social para el perfeccionamiento de modelos y la resolución de problemas (Apthorpe, 1997). De hecho, lo que está en juego en la persistente construcción del conocimiento como entidad o producto neutro no es sólo la justificación de las políticas públicas, sino además la propia noción de "consultoría". El proceso de conocer es público y disponible a nivel local, adaptado en sus diversas formas a las circunstancias particulares que lo hacen apropiado en lugar de "útil/ inútil" o "verdadero/ falso" en términos absolutos (Hobart, 1993b; Desai, 2006). Como en cierta forma revelan los resentimientos de algunos de los defensores de la "antropología aplicada" que contribuyen a la mencionada compilación de la Universidad de Querétaro, este argumento bastaría para obviar el papel de los consultores ("dueños de conocimiento") que se emplean en el mundo de las políticas. Por ello, el conocimiento ha de sustraerse de sus contextos de producción y comunidades epistémicas particulares para, vía su privatización y mercantilización mediante la consultoría, convertirse en un bien de consumo a ser acumulado, transferido o vendido a otras unidades de producción (Agudo Sanchíz, 2009). 
Aparte del continuado peso ideológico de la dicotomía entre teoría y práctica, existe un caso más de confluencia y retroalimentación entre desarrollo y crítica socio-antropológica. Hace ya tiempo que las entidades financieras internacionales y las instituciones dominantes de la política pública retomaron e instrumentalizaron las categorías del desarrollo "participativo" o "comunitario", promovidas en las décadas de 1980 y 1990 por activistas y voluntarios de organizaciones no gubernamentales (ONGs). El énfasis en el "conocimiento local" y desarrollo alternativo o "desde abajo", recomendado asimismo por antropólogos y críticos de otras disciplinas y ámbitos de trabajo (véase Chambers, 1983), se transmutó en la corresponsabilidad, la cogestión y la participación que muchos programas de política social demandan ahora de los ciudadanos. Esto ilustra algunos de los argumentos centrales del - pese a todo - importante trabajo de autores como Ferguson o Escobar: "Aunque el discurso ha pasado por una serie de cambios estructurales, la arquitectura de la formación discursiva establecida en el periodo 1945-55 ha permanecido sin cambios, permitiendo al discurso adaptarse a las nuevas condiciones" (Escobar, 1995; citado en Gardner y Lewis, 1996: 103-104). Después de todo, el desarrollo sigue estando dominado por principios de causalidad simple y su idea fundamental se mantiene: los programas sociales constituyen un intento de cambiar el mundo existente en ciertas formas predeterminadas e identificables. Conforme un paradigma de desarrollo humano fracasa y es sustituido por otro, lo que cambia son las ideas acerca de qué inversiones o aportaciones son las más apropiadas.

En este sentido, el subsidio ha sido reemplazado por el contrato con los ciudadanos participativos en modelos de política instrumentales y de corte simplista, donde los tipos y causas de la pobreza y la desigualdad son convenientemente soslayados. Las intervenciones del conocido programa mexicano Oportunidades, por ejemplo, consisten principalmente en transferencias monetarias condicionadas al cumplimiento de una serie de corresponsabilidades en salud, higiene, educación y alimentación mediante las que se espera que los beneficiarios "rompan el ciclo de pobreza" transmitido por ellos mismos de manera intergeneracional (véase SEDESOL, 2007: 3). De forma similar, ciertas intervenciones públicas en seguridad ciudadana, en las que también he participado como consultor-investigador, están guiadas por el enfoque de la "coproducción de la seguridad" (Ruiz y Vanderschueren, 2007), el cual busca implicar activamente a las comunidades en la prevención integral de las violencias y la delincuencia, mediante una relación más próxima y un trabajo de colaboración regular con las autoridades. En consonancia con los supuestos normativos sobre la "retirada del Estado" y el reforzamiento de la sociedad civil — con las ONGs como sustitutas de ciertas acciones gubernamentales-, la política pública resulta así ciudadanizada y concebida como un esfuerzo conjunto con fuertes tintes contractuales.

Lo que más me interesa recalcar, no obstante, es que la cooptación y normalización de la "participación" debe mucho a su propia ambigüedad y falta de profundización empírica mediante análisis de los contextos y relaciones sociales que implica —quién puede participar, cómo y por qué-. Una vez pasamos del discurso en los modelos y documentos de la política a las relaciones sociales, empezamos a ver cuáles de estas últimas — en donde - pueden realmente producir los órdenes 
moralizadores y regulativos implícitos en los programas de combate a la pobreza y seguridad ciudadana. Como Foucault diría, son dichas relaciones las que proporcionan el terreno concreto y cambiante para los efectos de tales programas. En ciertas localidades rurales de Chiapas, el cumplimiento de las corresponsabilidades de Oportunidades depende de jerarquías de conocimiento y relaciones comunitarias de poder, guiadas por la lógica del endeudamiento, que traducen el principio liberal del contrato a normas locales de "servicio a la comunidad" y beneficio colectivo (Agudo Sanchíz, 2011). En algunas colonias urbanas del norte de México, la transferencia del vigilantismo y la coerción oficiales a la ciudadanía, vía la "participación comunitaria" en la producción de la seguridad, está sujeta a conflictos y antagonismos locales cuyas demandas concomitantes de "toques de queda" pueden si acaso dar forma social a la militarizada jerga del "control natural de los accesos" y el "reforzamiento territorial" — según se expresa en un diagnóstico sobre prevención del delito auspiciado por el Banco Interamericano de Desarrollo (PBK, 2011: 14-16)-.

\section{La evaluación de impacto como proceso de socialización del consultor}

Las construcciones y modos de ordenamiento discursivo compartidos posibilitan lo que podríamos ver como una socialización del antropólogo en el seno de las comunidades profesionales de la política pública. Al mismo tiempo, por supuesto, dicha inducción a la toma de conciencia de las normas y valores de estas comunidades resulta necesaria por las lógicas diferencias epistemológicas y de objetivos entre la antropología y el desarrollo. Estas diferencias pueden ser en principio tan irreconciliables que den lugar a francas presiones y coerción sobre los consultores. Al inicio de la evaluación cualitativa de los efectos a largo plazo del Programa Oportunidades, por ejemplo, el coordinador nacional del mismo llegó a exigirnos a los antropólogos evaluadores que aportáramos "argumentos para defender el programa". La evaluación tenía lugar tras un año electoral sumamente tenso en el que se habían producido rumores de cancelación del programa con el cambio de administración federal, así como acusaciones al entonces gobernante Partido Acción Nacional de utilizar Oportunidades - y recursos de la Secretaría de Desarrollo Social (SEDESOL) - en beneficio propio durante la campaña previa a los comicios presidenciales de julio de 2006. Todo ello hizo imprescindible que, una vez finalizada la evaluación en 2008, los consultores acudiéramos a presentar los resultados de la misma ante una audiencia "VIP" compuesta por expertos de diversas nacionalidades, así como por representantes de dependencias gubernamentales e instituciones financieras internacionales (Agudo Sanchíz, 2012). Antes de iniciar las presentaciones, una funcionaria de alto rango del Programa Oportunidades abrió la sesión recordándonos a los consultores que aquello no se trataba de "investigación académica, sino de evaluación".

Lo último podía parecer sorprendente en vista de que el evento, celebrado en un elegante hotel al sur de la ciudad de México, había sido anunciado a bombo y platillo como un "seminario académico". Por otra parte, no obstante, ello era un recordatorio más de las funciones de legitimación y verificación que los mandos 
directivos de instituciones gubernamentales y de programas de política pública requieren de los científicos sociales, avalados por sus respectivas universidades o centros de investigación. Esta búsqueda de validación mediante la contratación de "expertos" puede tomar la forma de un mero requisito consistente en un estudio académico hecho al vapor y financiado a bajo costo (García Espejel, 2012b). Antes de que la Secretaría de Seguridad Pública fuese suprimida a fines de 2012 a propuesta del nuevo presidente de México - Enrique Peña Nieto-, una funcionaria me telefoneó para proponerme que coordinara una compilación de trabajos académicos a ser publicada en el plazo de dos meses bajo el sello de la dependencia. Tras declinar la invitación por varias razones, averigüé que varios colegas habían recibido propuestas similares.

Aparte de suponer un riesgo mucho menor en términos de devaluación de la antropología, por supuesto, el requerimiento oficial de una evaluación independiente del Programa Oportunidades en 2007-2008 obedeció al habitual mandato de la transparencia y la rendición de cuentas en la gestión pública. Lo realmente importante es que, a pesar de presentarse como "externas", tales evaluaciones son en realidad parte del propio proceso de producción de las políticas. A ello me refiero cuando digo que los investigadores evaluadores son socializados y disciplinados en lo que Stirrat (2000) denomina la "cultura de la consultoría", por lo que, aún sin compromisos o afiliaciones previos, estos nunca quedan completamente fuera de dicho proceso de la política².

Siguiendo en parte a Stirrat, en otro trabajo me he referido a esta cultura de la consultoría como un conjunto de ciertos principios del positivismo clásico —objetividad, racionalidad, impersonalidad-, a los cuales los evaluadores hemos de adherirnos como parte de una ideología compartida con la que hemos de lidiar de forma más o menos consciente (Agudo Sanchíz, 2012). Una de las primeras etapas de este proceso suele consistir en la elaboración y circulación de documentos vinculantes mediante los que las instituciones del desarrollo social definen los objetivos de sus consultores, incluyendo los rubros específicos que han de tener en cuenta en su trabajo de investigación: los imprescindibles "términos de referencia" y convenios de colaboración entre las instituciones involucradas en el diseño y evaluación de programas de política pública. En el cuerpo de los mismos o en sus anexos técnicos se establecen la estructura y el formato que deben seguir todos los documentos e informes producidos por los consultores, incluyendo los dos imprescindibles apartados del "resumen ejecutivo" — nadie está dispuesto a leer un reporte etnográfico de cien páginas - y el análisis que correlaciona de manera directa "fortalezas y oportunidades, debilidades y amenazas" (FODA): técnica que permite filtrar las múltiples experiencias de los consultores en el programa en cuestión para extraer una historia de impacto del mismo en forma sintética, produciendo un modelo de retroalimentación universal y uniforme que garantice información y aprendizaje para todos en

${ }^{2}$ Es precisamente este aprendizaje o socialización de tipo secundario lo que parecen exigirle a la academia y la universidad algunos de los partidarios de la antropología aplicada que contribuyen a la citada compilación de García Espejel (Garibay, 2012; Martínez Romero, 2012; Noroña, 2012; Vázquez-Mellado, 2012). 
todos los niveles. En este sentido y conforme a mi propia experiencia en Oportunidades, otro ejemplo especialmente exhaustivo de definición de los resultados de la evaluación se encontraba en la herramienta adicional de la "matriz de indicadores para resultados" del programa, la cual establecía en forma tabulada sus objetivos, resultados esperados y las relaciones anticipadas entre sus componentes, actividades y consecuencias: en el recuadro superior del diagrama podía leerse claramente el fin del programa: "Contribuir a la ruptura del ciclo intergeneracional de la pobreza extrema, favoreciendo el desarrollo de las capacidades en educación, salud y nutrición de las familias beneficiarias de Oportunidades" (Agudo Sanchíz, 2012: 58-60).

Como afirma Stirrat (2000: 42-43), la utilidad práctica de estos instrumentos puede resultar menos relevante que su capacidad estética para condensar los valores modernos de la objetividad y la impermeabilidad frente al carácter impredecible y caótico de la realidad. La concomitante estructuración linear y racional de esta última, sin embargo, está llamada a influir en el diseño de la evaluación de impacto del programa, ya que guía la selección de información considerada relevante. Esto no significó que los evaluadores careciéramos de libertad para diseñar nuestra propia metodología, la cual incluyó una muestra analítica que guiaría la selección de un número fijo de grupos domésticos receptores y no receptores de transferencias del programa, indígenas y no indígenas - en idénticas proporciones-, así como de los miembros de dichos hogares considerados prioritarios para las entrevistas. Estas últimas abarcaban una serie de temas y preguntas que hacían hincapié en los cambios acaecidos en salud, educación y trabajo en un lapso de diez años. Más aún, tuvimos la posibilidad de innovar lo suficiente como para introducir en la evaluación temas que, en un principio, añadían una complejidad no solicitada por la coordinación nacional del programa. Así, nos propusimos dirigir la mirada hacia la calidad de los servicios de educación y salud — cuya demanda estimula Oportunidades- y la cobertura y operación del propio programa en las regiones estudiadas en el norte - Sonora y Chihuahua - y sur de México - Oaxaca y Chiapas-, empleando como punto de partida la hipótesis de que los grupos indígenas receptores de los apoyos de Oportunidades obtenían impactos menos favorables que los no indígenas.

Lo anterior suscitó desconfianza e incluso una franca resistencia por parte de ciertos actores institucionales de Oportunidades, si bien otros funcionarios influyentes del programa y los integrantes de un grupo de asesores externos de la evaluación (GAE) se mostraron más favorables a nuestras propuestas. Ésta fue la primera fase de confrontación en la "arena" de la evaluación entre individuos y grupos que, a la manera de stakeholders - partes interesadas-, teníamos algún provecho u objetivo particular en la misma. La inclusión de la espinosa hipótesis del "impacto diferencial" de Oportunidades en poblaciones indígenas, así como la evaluación de la cobertura y operación del propio programa, podía verse como una manera de incrementar capital político con base en la demostración de transparencia y legitimidad por parte de actores institucionales opuestos a "la perpetuación de las desigualdades más graves del país". Asimismo, en caso de producirse resultados negativos en nuestros diagnósticos sobre los servicios públicos, los funcionarios del programa se deslindarían, exigiendo a los evaluadores dirigir sus recomendaciones 
a las instituciones responsables de los sectores educativos y de salud. Desde el punto de vista de los antropólogos consultores, los resultados de las innovaciones aportadas a la evaluación cualitativa podrían proporcionar material para posteriores investigaciones y publicaciones académicas. Al menos desde mi perspectiva, también se abría la posibilidad de interrogar críticamente la despolitizada teoría causal de la "ruptura del ciclo intergeneracional de la pobreza extrema" — la cual aparece así como resultado de las propias prácticas de los pobres-, al igual que otros conceptos recogidos en los documentos de Oportunidades. En las fases subsiguientes de la evaluación, no obstante, empezarían a aflorar las limitaciones impuestas a la posible tensión creativa emanada del debate sobre significados y la construcción compartida de conocimiento en torno a los impactos de Oportunidades. Los problemas de representación que pudiéramos haber ocasionado los evaluadores antropológicos tendieron a resolverse mediante un sistemático trabajo conceptual que llevaría al programa de "regreso al texto" y a sus ideas rectoras, recordándonos que una "buena evaluación" es aquélla que sostiene al modelo de la política (Mosse, 2005: 157-158, 179).

$\mathrm{Al}$ enfocarnos en temas que iban más allá de la mera evaluación de "impactos", empezamos a hacer visibles ciertos eventos y relaciones que caracterizaban a la transferencia de recursos de Oportunidades en las regiones y localidades estudiadas. En clara contravención de la pulcritud tabulada de la matriz de resultados del programa, nuestros primeros informes preliminares recogerían análisis sobre las "prácticas de simulación y complicidad" mediante las que prestadores de servicios y beneficiarios daban un contenido y una lógica distintos a las corresponsabilidades (véase Agudo Sanchíz, 2011). La intervención de intereses y actores locales contradecía asimismo la representación de "transparencia" del programa y su proclama de evitar interferencias en la entrega de sus beneficios. Finalmente, nuestra investigación sobre la operación y cobertura de Oportunidades arrojó irregularidades como la incorporación al programa de grupos enteros de hogares sin la encuesta previa de certificación que había de realizarse casa por casa.

Enseguida nos vimos expuestos a una insistente serie de críticas y recomendaciones realizadas desde varios frentes, las cuales enfatizaban la necesidad de evitar emitir "demasiadas debilidades" con respecto al programa (Agudo Sanchíz, 2012: 66-72). En un documento emitido por la propia SEDESOL se nos informaba a los evaluadores que "no es necesario remarcar palabras como 'improvisación', 'favores', 'complicidades', etc. ... No es necesario enfatizar que existe un conjunto de 'prácticas de simulación y complicidad', basta señalar que existe un conjunto de 'practicas [sic] que han hecho posible la permanencia de las familias en un contexto de extrema fragilidad de las instituciones...". Junto con el empleo de tales giros eufemísticos, otras sugerencias en el mismo documento nos urgían a ser "específicos" y "claros" para vincular directamente la información reportada con las recomendaciones que habríamos de exponer en el Análisis FODA: "erradicar las prácticas de simulación" no sería una recomendación concreta para el programa.

Otras técnicas para disciplinar nuestro estilo de expresión consistían en señalar los términos políticamente incorrectos o problemáticos como meros "adjetivos 
que sólo llaman la atención, pero no aportan información valiosa" o, incluso, como "juicios de valor". La recomendación más frecuente en este caso era "justificar", "sustentar" u "operacionalizar" conceptos como "discriminación" o "exclusión" con base en la evidencia empírica. Como especificaba un documento del Consejo Nacional de Evaluación de la Política de Desarrollo Social (CONEVAL) —el organismo público descentralizado que se encarga de normar y dar seguimiento a las evaluaciones de programas sociales en México - " "cuando se señala un acto infantilizador, discriminatorio o grosero por parte del personal de salud hacia la población indígena, se requiere de una descripción de las diferencias o similitudes respecto de lo observado con la población pobre no indígena". Cualquiera podría concordar con la necesidad de describir lo observado antes de llegar a conclusiones, si bien este criterio fue aplicado de manera muy desigual. Mientras que unas veces se nos pedía que sustentáramos el empleo de términos como "discriminación" con base en las prácticas observadas en contextos específicos, otras se nos conminaba a ofrecer definiciones universales de dichos términos, las cuales habrían de coincidir en todos los documentos producidos por los evaluadores tras el trabajo de investigación en regiones muy diversas.

Junto con la autoría individual que denotaban los "juicios de valor", dudas y opiniones personales, la contingencia y la heterogeneidad habrían así de ser excluidos de los informes finales de la evaluación. Otro documento procedente del propio Programa Oportunidades sintetizaba de manera particularmente clara lo que se requería de los evaluadores: "Presentar los resultados más de tipo [en forma de] diagnóstico, aquéllos [son] más bien de tipo descriptivo". Se nos recordaba así uno de los principales obstáculos para el empleo de etnógrafos en las políticas públicas: el lenguaje de estas últimas no busca ser descriptivo, sino prescriptivo, ya que no necesitan informes que hablen de algo "como realmente es", sino evaluaciones que digan claramente "qué es lo que se necesita" —y aquí el lenguaje "claro" viene dado por el hecho de excluir ciertos detalles del informe (Apthorpe, 1997) - No obstante, nuestro reto como antropólogos debería consistir de hecho en "unir" esa aparente brecha entre lo normativo y lo descriptivo o, de manera más precisa, demostrar cómo muchos rasgos del orden jurídico de la política pública y el orden social local no se explican de manera aislada (cfr. Azuela, 2011). Los órdenes locales que describíamos en nuestros informes estaban impregnados de normatividades sobre las que, precisamente, descansaba la condición de posibilidad del cumplimiento de las obligaciones del programa, aunque éste apareciera "distorsionado" en las comunidades estudiadas.

Sin embargo, estas disquisiciones no eran compatibles con la urgencia de que los evaluadores de Oportunidades aprendieran el arte y la estética de representar eventos en términos de modelos normativos, excluyendo las contingencias de la política mediante apelaciones a la imparcialidad y contribuyendo a la permanencia de una idea particular de política social. Uno de mis colegas evaluadores me confió que "no pensé que para eso nos hubieran contratado. Siento que hay que matizar tanto las afirmaciones que casi se vuelven en negaciones. Así que no me veo en un futuro en esta misma tarea". Pasamos por un intenso y agotador proceso de discusión, 
corrección y refinación de sucesivas versiones de nuestros informes, cada vez más prolongado y alejado del trabajo de campo. Los comentarios y recomendaciones que recibíamos se dirigían al estilo de expresión empleado en nuestros textos, incrementando mi sensación de que la "calidad" de la evaluación dependía de la de los documentos que producíamos; éstos parecían convertirse en fines en sí mismos mas que en medios para dar a conocer los logros y debilidades del programa y actuar sobre ellos. No obstante, el género de los textos de la política social y sus evaluaciones funciona como una forma de poder y, por lo tanto, sí tiene un fin concreto que a menudo consiste en persuadir antes que informar (Apthorpe, 1997).

Naturalmente, el poder interpretativo de los documentos de la evaluación también depende de cómo y por quiénes sean leídos, lo cual incluye el estatus de sus detractores y defensores en la arena más amplia de la política social -implicando a funcionarios públicos, académicos y otros consultores de prestigio reconocidoLos expertos del GAE que "asesoraban" nuestra evaluación podían tener - y permitirse expresar - una visión crítica del programa, de la cual los consultores podíamos derivar una cierta perspectiva de apoyo. Así sucedió en mi caso cuando una experta en educación destacó los aspectos positivos de la metodología y los resultados que dejaban ver mi informe preliminar sobre la investigación que había coordinado en Chiapas. La socialización del consultor también tiene lugar mediante la interacción con diversos actores significativos: la escritura de mi documento técnico final estuvo influida por las perspectivas de esta asesora y por mi búsqueda implícita de su aceptación frente a los miembros del CONEVAL — antropólogos entre ellos- que habían criticado mi texto original por su falta de consistencia.

Las correcciones hechas por el CONEVAL, sin embargo, habían de ser incorporadas por ley a los documentos finales de la evaluación - los que sí se harían públicos-, mientras que las recomendaciones del GAE y de los representantes del programa tenían en principio el carácter de insumos para la calidad de los mismos. Todos estos actores podían en cualquier caso contribuir a cierta diversidad de voces - aunadas a las de los antropólogos evaluadores - que reflejaran las jerarquías, desigualdades y contradicciones inherentes a la arena de la política pública. Precisamente por todo ello, y por la inexistencia de áreas de centralidad e imparcialidad, los resultados de la evaluación habían de ser finalmente presentados conforme a una "coherencia" y una "consistencia" que dejaran el menor espacio posible para las interpretaciones contrastantes a las que de todas formas estaban sujetos.

Fue en esta última etapa de la evaluación cuando las herramientas mencionadas al principio, en especial la matriz de indicadores para resultados, revelaron su utilidad de forma particularmente clara. El mencionado informe preliminar sobre Chiapas necesitaba sin duda reestructurarse y revisarse conforme a un análisis más cuidadoso del material recopilado en campo, aunque la crítica recibida por el mismo exigía fidelidad a los formatos de presentación y al modelo de impacto del programa:

El informe de Chiapas rompe con el formato acordado de presentación... No da seguimiento a los objetivos, preguntas e hipótesis que se plantean en el anexo 1 [del convenio firmado con SEDESOL]... Por lo tanto, se sugiere al equipo de 
investigación en Chiapas que respete el esquema propuesto en la Guía y ordene los resultados de esa manera, a fin de que se cumpla con la evaluación propuesta. También es necesario que centre su discusión en las preguntas fundamentales que guían la evaluación. ¿Podemos evidenciar que el diseño y la operación del Programa, en el largo plazo, contribuye a romper el ciclo de transmisión intergeneracional de la pobreza? (citado en Agudo Sanchíz, 2012: 73; énfasis añadido).

En una suerte de desvanecimiento de fronteras entre lo normativo y lo descriptivo, el diseño del programa llegaba a invocarse como la experiencia del mismo. En los documentos resultantes del estudio de impacto habría de separarse el conocimiento sobre el programa de sus implicaciones prácticas y traducirlo a prescripciones. La comentadora de mi texto sobre Chiapas — una antropóloga del CONEVAL con experiencia de investigación en la región - continuaba recordándome la teoría causal de Oportunidades: "Entre líneas, el lector podría concluir que el Programa en Chiapas, de acuerdo a los resultados que expone, no rompe con dicha transmisión intergeneracional de la pobreza". De hecho, el modelo se volvía tan importante que debía reinstaurarse como propósito clave del programa. Una académica que fungía como lectora externa llegó a criticar el tono excesivamente negativo de otro de nuestros informes, advirtiendo a su autor que habría de ser más cuidadoso en sus apreciaciones sobre la cobertura y operación del Programa Oportunidades, ya que éste "cuenta con uno de los mejores modelos de política social que conozco". El escrutinio del programa se dirigía así a su teoría más que a su práctica, el proceso de la política volvía a replegarse sobre sí mismo y la evaluación regresaba de la experiencia del trabajo de campo a la adaptación a las matrices de resultados.

Una vez blindado el programa contra cualquier confrontación seria con la realidad, o al menos con la información que cuestionaba sus vínculos causales entre diseño e implementación, faltaba otro paso clave para consensuar una versión de la evaluación convincente que pudiera transformarse en un "artículo de consumo ampliamente compartido" (Phillips y Edwards, 2000). Ahora todas las novedades que los antropólogos habíamos logrado introducir en la evaluación se convertían en "temas adicionales" o secundarios, los cuales debían excluirse del análisis FODA y trabajarse de alguna forma en un aparte para "lograr cierta homogeneización entre los análisis". Esta fue de hecho la palabra clave, repetida una y otra vez en una especie de hoja de ruta proporcionada por el programa y respaldada por el CONEVAL: habríamos de homogeneizar el índice de todos los documentos, la estructura y extensión de los resúmenes ejecutivos, la presentación de las citas o testimonios relevantes, el abordaje de los "grandes temas" y el análisis FODA (Agudo Sanchíz, 2012: 74). Oportunidades también habría de preservarse de las posibles interpretaciones de una audiencia diversa y no siempre predecible; el programa adquiría asimismo realidad social mediante la uniformidad de puntos de vista, la consistencia entre interpretaciones y la resolución de desacuerdos sobre sus "impactos positivos".

En este último sentido, aun quedaban otros flecos que recortar y resistencias de última hora que vencer. Los resultados de las pequeñas muestras seleccionadas para la evaluación, en especial en varias regiones del sur del país, arrojaban una prolongación de trayectorias escolares femeninas, lo cual fue inmediatamente recibido 
como un dato muy positivo del "impacto educativo" de Oportunidades. Una vez la evidencia empírica se convierte en una cuestión de consenso, resulta más arduo introducir argumentos discordantes en la construcción colectiva de los efectos de un programa. A pesar de ello, hice un último intento de recomendar cierta cautela sobre los resultados relativos a la discriminación educativa en contra de las niñas. Propuse la hipótesis, basada en mis resultados de investigación, de que las becas de Oportunidades podían posponer meramente el abandono escolar de las jóvenes hasta la conclusión de la educación secundaria, especialmente en localidades rurales con presencia de centros de dicho nivel educativo. Otros escenarios podían darse donde sólo existiese cercanía a escuelas primarias y/o donde las jóvenes hubiesen de desplazarse a otras localidades o incluso ciudades para estudiar el bachillerato, con la posible oposición de sus familias. Se trataba, en suma, de matizar la univocidad y la causalidad simple del modelo del programa mediante el reconocimiento de los múltiples factores con los que interactúan sus becas escolares. De hecho, la mayor permanencia escolar de los niños era a lo sumo un resultado directo de dichas becas, cuyo impacto según el objetivo del aumento de capacidades y la ruptura de la pobreza intergeneracional era difícil establecer debido a la mala calidad de la educación y la ausencia de oportunidades laborales. En una de las últimas reuniones con integrantes del GAE y del CONEVAL, no obstante, se me conminó de manera vigorosa a excluir la proposición de la discriminación educativa de mi informe final ${ }^{3}$.

Parte del correctivo provino de alguien perteneciente al propio equipo de antropólogos evaluadores. Pero yo tampoco estaba libre de actitudes intransigentes. Al inicio de la evaluación había argumentado a favor de privilegiar la "lengua materna distinta del español" como criterio bajo el cual habría de considerarse "indígenas" a comunidades y familias, cerrándome a otros principios más abiertos e interpretativos planteados por colegas antropólogos e incluso por algunos asesores de la evaluación - por ejemplo, auto-adscripción y adscripción por parte de otros grupos- Esto fue tanto un error personal como una postura influida por las mismas condiciones estructurales y discursivas que pesaban sobre mis compañeros consultores: quise presentar la inclusión de "la variable indígena" conforme a los criterios de las bases de datos empleadas por el propio programa, apelando así de manera implícita a los funcionarios de Oportunidades en busca de aprobación para continuar con la investigación. En 2007-2008, con apenas 35 años de edad y menos de un lustro de experiencia en consultoría, fui operacionalmente marginal en la arena de la evaluación de Oportunidades; pero también contribuí a la reducción de realidades complejas a "variables" y categorías objetivadas, así como a las representaciones de la realidad de los impactos del programa.

\section{Reflexiones finales}

Casi cada vez que se habla de la antropología de —y en — las políticas públicas, resurgen ciertas suposiciones y dicotomías que, desde posiciones contrastantes y

${ }^{3}$ Los documentos resultantes de la evaluación de Oportunidades en 2008 pueden aún consultarse en: http://lanic.utexas.edu/project/etext/oportunidades/. 
mediante versiones diversas, reproducen la separación más general entre representación y realidad. Así, los exponentes de cierta vertiente antropológica crítica del desarrollo asumen de manera implícita efectos — casi siempre funestos - a partir de diseños o discursos, mientras que los defensores de la incorporación de la antropología a la consultoría no dejan de hablar de la "aplicación práctica" de la disciplina; de este modo, todos contribuyen al mantenimiento de la autoridad de la planificación racional. La postura de los últimos es, si cabe, más contradictoria, ya que no queda claro en absoluto cómo podría aplicarse una disciplina - la suya - a la que atacan por excesivamente abstracta y divorciada de las necesidades del mundo real y sus mercados laborales. De forma aún más incomprensible e incluso contraria a sus intereses, afirman categóricamente que:

[e]n México, los antropólogos no están preparados para hacer consultoría, es decir, antropología aplicada, que implica, en comparación con la académica: Tiempos más cortos ... Metodología no antropológica, variedad de técnicas ... Habilidades para el trabajo en equipo ... Actitud de servicio y humildad [y, finalmente,] Relación con la autoridad de reconocimiento y respeto. Acatar normas (Vázquez-Mellado, 2012: 145).

A juzgar por nuestro uso de perspectivas filosóficas sobre el significado de la pobreza o la adopción de marcos sociológicos para el análisis de la vulnerabilidad social, no veo que ni en mi formación antropológica ni en la de mis colegas consultores en Oportunidades - al mismo tiempo académicos como yo- haya habido algo que necesariamente nos impidiese tomar préstamos de otros enfoques y disciplinas. Esto es lo que hacen también muchos otros antropólogos en su búsqueda de análisis alternativos que resulten útiles para evaluar un programa de política social o proponer soluciones a problemas en otros ámbitos. Tampoco me parece que los tiempos más cortos y las habilidades para el trabajo en equipo estén excluidos de lo que Vázquez-Mellado llama antropología académica, en especial por la carga de horas de docencia con la que muchos hemos de combinar nuestras investigaciones, así como por la creciente propensión de ciertas instituciones de ciencia y tecnología a financiar proyectos colectivos e incluso interdisciplinarios. En cuanto a la supuesta ausencia de actitud de servicio, humildad y reconocimiento respetuoso en las universidades y centros de investigación, sólo se me ocurre compadecer a la autora por la experiencia negativa que, al parecer, supuso su paso por la institución en la que cursó su licenciatura en antropología. Después de lo descrito aquí, no me parece que las desigualdades, jerarquías y relaciones de poder sean exclusivas del ámbito académico, a menos que se equipare el "reconocimiento" y el "respeto" con la autodisciplina y la aquiescencia a generar legitimidad y apoyos para las políticas públicas.

Esa bifurcación del mundo de la antropología en ámbitos aplicados y académicos, así como la tendencia general a asumir lo irreconciliable y antagónico de la disciplina respecto de las políticas públicas, impiden ver las diferencias pero también las coincidencias reales que existen entre la primera y las segundas. Después de todo, tanto la distancia y el recelo de unos antropólogos como el compromiso y la 
participación directa de otros pueden compartir una incómoda conciencia de cierta proximidad: la antropología — sin adjetivos - y el desarrollo emplean modos de ordenamiento discursivo y lógicas similares en sus respectivos intentos por resolver la tensión experimentada entre el conocimiento generalizable y la especificidad de situaciones e ideas particulares (Yarrow, 2008). Lidiar con dicha tensión fue de hecho uno de los principales cometidos de los consultores y asesores académicos de la evaluación de Oportunidades, quienes además hubimos de mediar entre el conocimiento de nuestra disciplina y las representaciones del desarrollo.

Tensión y mediación son propias de un proceso de confrontaciones y negociaciones caracterizado por la polifonía, las identidades múltiples y la reflexividad sobre el propio papel desempeñado en la construcción colectiva del conocimiento. Guiados por una suerte de vocación para cambiar al desarrollo "desde dentro", algunos antropólogos han argumentado que, empleadas de manera consciente, las perspectivas "posmodernas" de la disciplina sobre estos procesos pueden reorientar la práctica de la política pública y retroalimentar agendas prácticas de manera fructífera (Gardner y Lewis, 1996; Eyben, 2000). Sin embargo, también hay aquí ciertas paradojas. Por un lado, lo que hace posible el diseño y la evaluación de un programa es precisamente la vieja suposición empirista de una realidad aprehensible - y predecible - sin la mediación de valores, por lo que la idea de una "consultoría posmoderna" resultaría en este sentido una contradicción flagrante (Stirrat, 2000: 40). Por otro lado, la tensión emanada de tendencias contrarias y valores e identidades contrastantes ciertamente existe, aunque su resolución, mediante las distintas técnicas discutidas aquí, tiende al sostenimiento de construcciones auto-referenciales de impactos y resultados - mostrando un inmanentismo reminiscente de posturas posmodernas extremas que enfatizan comunidades interpretativas cerradas, caracterizadas por discursos compartidos con reglas propias y sin anclaje referencial alguno en el mundo externo-. En este último sentido, muchas consultorías para la evaluación de programas sociales serían inevitable al tiempo que inconfesablemente "posmodernas": de ahí que se revistan públicamente con ciertos ropajes seleccionados de la modernidad clásica —objetividad, racionalidad, univocidad - para ocultar lo que las constituye y evitar así poner en peligro las predicciones de los modelos de la política que buscan corroborar (Agudo Sanchíz, 2012). Podría invocarse aquí la metáfora, empleada por la teoría del actor-red, de la "caja negra" que ha de permanecer cerrada para no revelar los procesos y mecanismos por los que se ha llegado al producto final (cfr. Mosse, 2005: 179). De ahí la frustración experimentada por autores como Van den Berg y Quarles van Ufford (2005), quienes también anuncian la importancia creativa y las útiles consecuencias prácticas del reconocimiento de las inconsistencias y la "marginalidad" de las prácticas del desarrollo, pero, en realidad, terminan señalando de manera un tanto pesimista los resultados del silenciamiento de dichas inconsistencias y marginalidad.

Por todo ello, el reconocimiento de la naturaleza negociada de las evaluaciones resulta incompatible con la epistemología y las nociones de causalidad dominantes en las políticas, vinculadas a su permanente necesidad de legitimación. Hay, sin embargo, excepciones fructíferas como el trabajo de Phillips y Edwards (2000). 
Estos consultores-autores antropológicos adoptaron la estrategia de incluir a los operadores locales de un proyecto "participativo" de desarrollo urbano en una evaluación externa de impacto, planeada de manera vertical por los - muy poco satisfechos - expertos de la institución patrocinadora. Al tener en cuenta las experiencias y perspectivas del inicialmente excluido y renuente personal de planta en el informe de la evaluación, ésta fue generalmente reconocida como "justa" y el proyecto - anteriormente bajo amenaza de cancelación - fue continuado. Este caso ejemplifica cómo la incorporación consciente de la polifonía puede rescatar a un programa social de las tensiones y conflictos derivados de la jerarquía y el unilateralismo.

En cualquier caso, es preciso discutir claramente las limitaciones y demandas contradictorias con las que diversos partícipes han de lidiar en la arena de las políticas públicas. Asimismo, necesitamos tener en cuenta que los problemas derivados de la desarticulación entre la experiencia y los modelos racionalizadores de las políticas intentan solucionarse mediante un continuado trabajo conceptual, cuyos esfuerzos van destinados a mantener los programas sociales como teorías y representaciones (Mosse, 2005). En otras palabras, son las prácticas sociales las que realmente sostienen a los modelos de las políticas, aún cuando se siga asumiendo que los segundos generan las primeras. Más allá de las dicotomías y relaciones jerárquicas entre teoría y práctica sostenidas por unos y otros, es necesario reflexionar seriamente sobre este proceso y sobre lo que nos motiva a todos los que participamos en él. Dicha reflexión crítica es difícil sin la participación directa y el compromiso constructivo que implican la cercanía con la política administrativa de la práctica del desarrollo social.

\section{Referencias bibliográficas}

AGUDO SANCHÍZ, Alejandro

"Conocimiento, lenguaje, poder e intermediación. Perspectivas contemporáneas en la antropología de las políticas públicas". Estudios Sociológicos, XXVII (79): 63-110.

2011 "Mejoras privadas, beneficios colectivos: la producción y subversión de regímenes globales de política social en Chiapas", en A. Agudo Sanchíz y M. Estrada Saavedra (eds.), (Trans)formaciones del Estado en los márgenes de Latinoamérica. Imaginarios alternativos, aparatos inacabados y espacios transnacionales. México: UIA/ COLMEX, 231-283.

2012 "Consultorías (pos)modernas. La mímica del positivismo y la construcción del conocimiento en la evaluación de programas sociales". Estudios Sociológicos, XXX (88): 45-86.

AGUIRRE BELTRÁN, Gonzalo

1992 [1976] "El indigenismo y la antropología comprometida", en G. Aguirre Beltrán, Obra Antropológica (Tomo XI: Obra Polémica). México: Universidad Veracruzana / INI / Gobierno del Estado de Veracruz / FCE, 181-216.

APTHORPE, Raymond

1997 "Writing Development Policy and Policy Analysis Plain or Clear: On Language, Genre and Power", en C. Shore y S. Wright (eds.), Anthropology of Policy: Critical Perspectives on Governance and Power. Londres y Nueva York: Routledge, 42-58. 
AZUELA, Antonio

2011 “Algo que no sepamos? Casi todo”. Nexos, 398 (febrero): 13-15.

CHAMBERS, Robert

1983 Rural Development: Putting the Last First. Nueva York: John Wiley.

DESAI, Bina

2006 "Inside Out: Rationalizing Practices and Representations in Agricultural Development Projects", en D. Lewis y D. Mosse (eds.), Development Brokers and Translators. The Ethnography of Aid and Agencies. Bloomfield, Connecticut: Kumarian Press, 173-193.

ESCOBAR, Arturo

1995 Encountering Development: the making and unmaking of the Third World. Princeton: Princeton University Press.

EYBEN, Rosalind

2000 "Development and Anthropology: A View from Inside the Agency". Critique of Anthropology, 20 (1): 7-14.

FERGUSON, James

1994 The anti-politics machine: development, de-politicisation and bureaucratic power in Lesotho. Minneapolis: University of Minnesota Press.

1997 "Anthropology and its evil twin: development in the constitution of a discipline", en F. Cooper y R. Packard (eds.), International Development and the Social Sciencies. Essays on the History and Politics of Knowledge. Berkeley: University of California Press, 140-172.

GAMIO, Manuel

1979 [1922] La población del valle de Teotihuacan. México: Instituto Nacional Indigenista.

GARCÍA ESPEJEL, Alberto (Coord.)

2012a Antropología y Consultoría. Una vertiente para el ejercicio profesional de los antropólogos. Querétaro, México: Universidad Autónoma de Querétaro.

2012b "Tres vertientes de aplicación de la antropología”, en A. García Espejel (coord.), Antropología y Consultoría. Una vertiente para el ejercicio profesional de los antropólogos. Querétaro, México: Universidad Autónoma de Querétaro, 147-174.

GARDNER, Katy; LEWIS, David

1996 Anthropology, Development and the Post-Modern Challenge. Londres: Pluto Press. (Edición en español: Antropología, desarrollo y el desafio posmoderno. Zinacantepec, Estado de México: El Colegio Mexiquense, 2003).

GARIBAY, Ricardo María

2012 "La utilidad de la antropología y la inutilidad de los antropólogos", en A. García Espejel (coord.), Antropología y Consultoría. Una vertiente para el ejercicio profesional de los antropólogos. Querétaro, México: Universidad Autónoma de Querétaro, 219-232. 
GLEDHILL, John

2000 El Poder y sus Disfraces. Perspectivas Antropológicas de la Política. Barcelona: Edicions Bellaterra.

GONZÁLEZ, Roberto

2007 "Towards mercenary anthropology? The new US Army counterinsurgency manual FM 3-24 and the military-anthropology complex". Anthropology Today, 23 (3): 14-19.

GONZÁLEZ CASANOVA, Pablo

1965 La democracia en México. México: Ediciones ERA.

HOBART, Mark (Ed.)

1993a An Anthropological Critique of Development. Londres y Nueva York: Routledge.

1993b "Introduction: the growth of ignorance?", en M. Hobart (ed.), An Anthropological Critique of Development. Londres y Nueva York: Routledge, 1-30.

HOUTMAN, Gustaaf

2006 "Double or Quits". Anthropology Today, 22 (6): 1-3.

KUPER, Adam

2005 "Alternative Histories of British Social Anthropology". Social Anthropology, 13 (1): 47-64.

LAGARDE, Marcela

1979 "El indio de la antropología mexicana". Historia y Sociedad, 21: 75-93.

LI, Tania Murray

1999 "Compromising power: development, culture, and rule in Indonesia". Cultural Anthropology, 14 (3): 295-322.

MARTÍNEZ ROMERO, Griselda

2012 "Cómo llegar a ser consultor sin morir en el intento de ser antropólogo", en A. García Espejel (coord.), Antropología y Consultoría. Una vertiente para el ejercicio profesional de los antropólogos. Querétaro, México: Universidad Autónoma de Querétaro, 41-53.

MITCHELL, Timothy

2002 Rule of Experts. Egypt, Techno-politics, Modernity. Berkeley: University of California Press.

MOSSE, David

2005 Cultivating Development. An Ethnography of Aid Policy and Practice. Londres: Pluto Press.

MOSSE, David; LEWIS, David (Eds.)

2005 The Aid Effect. Giving and Governing in International Development, Londres: Pluto Press 
NOROÑA, Arturo

2012 "No, la verdad es que no sé lo que hace un antropólogo", en A. García Espejel (coord.), Antropología y Consultoría. Una vertiente para el ejercicio profesional de los antropólogos. Querétaro, México: Universidad Autónoma de Querétaro, 115-132.

NUGENT, David; VINCENT, Joan (Eds.)

2004 A Companion to the Anthropology of Politics. Oxford: Blackwell.

PBK (PUBLIKA CONSULTING)

2011 Diagnóstico y línea de base para evaluación de impacto CPTED y de prevención situacional. Santiago, Chile: PBK.

PHILLIPS, Sue; EDWARDS, Richard

2000 "Development, Impact Assessment and the Praise Culture". Critique of Anthropology, 20 (1): 47-66.

ROHDE, David

2007 "Recluta Ejército a antropólogos como asesores". Diario Reforma, 12 de octubre.

RUIZ, Juan Carlos; VANDERSCHUEREN, Franz (Eds.)

2007 Consolidación de los gobiernos locales en Seguridad Ciudadana: formación y prácticas. Madrid: URB-AL/ Europe Aid Cooperation Office.

SACHS, Wolfgang (Ed.)

1992 The development dictionary: a guide to knowledge as power. Londres: Zed Books.

SEDESOL (Secretaría de Desarrollo Social)

2007 "Reglas de Operación del Programa de Desarrollo Humano Oportunidades, para el ejercicio fiscal 2008". Texto completo: http://www.oportunidades.gob.mx/Portal/ wb/Web/reglas_de_operacion_pdf0, (última consulta 14 de abril de 2013).

SHARMA, Aradhana; GUPTA, Akhil (Eds.)

2006 The Anthropology of the State. A Reader. Oxford: Blackwell.

SHORE, Cris; WRIGHT, Susan

1997 "Policy: A new field of anthropology", en C. Shore y S. Wright (eds.), Anthropology of Policy: Critical Perspectives on Governance and Power. Londres y Nueva York: Routledge, 3-39.

STIRRAT, Roderick L.

2000 "Cultures of Consultancy". Critique of Anthropology, 20 (1): 31-46.

VAN DEN BERG, Rob; QUARLES VAN UFFORD, Philip

2005 "Disjuncture and Marginality. Towards a New Approach to Development Practice", en D. Mosse y D. Lewis (eds.), The Aid Effect. Giving and Governing in International Development. Londres: Pluto Press, 196-212. 
VÁZQUEZ-MELLADO, Rosa María

2012 “Por qué vale la pena ser antropóloga”, en A. García Espejel (coord.), Antropología y Consultoría. Una vertiente para el ejercicio profesional de los antropólogos. Querétaro, México: Universidad Autónoma de Querétaro, 133-146.

VIOLA, Andreu (Comp.)

2000 Antropología del desarrollo. Teorías y estudios etnográficos en América Latina. Barcelona: Paidós Studio.

WARMAN, Arturo; NOLASCO, Margarita; BONFIL, Guillermo; et al.

1970 De eso que llaman antropología mexicana. México: Nuestro Tiempo.

YARROW, Thomas

2008 "Paired Opposites: Dualism in Development and Anthropology". Critique of Anthropology, 28 (4): 426-445. 